\title{
STANDARD MODELS FOR SOME COMMUTING MULTIOPERATORS
}

\author{
V. MÜLLER AND F.-H. VASILESCU \\ (Communicated by Palle E. T. Jorgensen)
}

\begin{abstract}
An analogue to the Sz.-Nagy-Foias dilation theory is presented for several commuting operators on a Hilbert space.
\end{abstract}

The dilation theory of Hilbert space contractions, a reference of which is the excellent monograph [9], has not yet found a complete counterpart valid for several (commuting) Hilbert space operators. Apart from the results of this type already contained in [9] and other results of general character (i.e., Arveson's or Stinespring's extension theorem), there are not too many contributions specialized to the multiparameter dilation theory. Among these contributions, we quote the works $[7,3-5,10]$, which are closer to our topics.

The aim of this paper is to analyse some positivity conditions for commuting multioperators, which ensure the unitary equivalence of these objects to some standard models consisting of backwards multishifts. We emphasize, in particular, a situation that seems to satisfy the requirements of an appropriate extension for the dilation theory of Hilbert space contractions and that exploits the results already obtained in $[4,10]$.

Our finer methods also imply better statements of some assertions from [10] and provide an answer to Question 4.5 from [5].

Let $n \geq 1$ be an integer, and let $\mathbf{Z}_{+}^{n}$ be the set of all $n$-tuples of nonnegative integers (i.e., the multi-indices of length $n$ ). If $\alpha=\left(\alpha_{1}, \ldots, \alpha_{n}\right) \in \mathbf{Z}_{+}^{n}$, we set, as usual, $|\alpha|=\alpha_{1}+\cdots+\alpha_{n}$ and $\alpha !=\alpha_{1} ! \cdots \alpha_{n} !$. For $\alpha, \beta \in \mathbf{Z}_{+}^{n}$ we write $\alpha+\beta=\left(\alpha_{1}+\beta_{1}, \ldots, \alpha_{n}+\beta_{n}\right)$, and $\alpha \leq \beta$ whenever $\alpha_{i} \leq \beta_{i}(i=1, \ldots, n)$.

Let $H$ be a Hilbert space, and let $\mathscr{L}(H)$ be the algebra of all bounded linear operators on $H$. An element $T=\left(T_{1}, \ldots, T_{n}\right) \in \mathscr{L}(H)^{n}$ such that $T_{1}, \ldots, T_{n}$ mutually commute will be designated as a commuting multioperator (briefly, a c.m.).

Let $T \in \mathscr{L}(H)^{n}$ be a c.m. We define the operator $M_{T}: \mathscr{L}(H) \rightarrow \mathscr{L}(H)$ by the formula

$$
M_{T}(X)=\sum_{i=1}^{n} T_{i}^{*} X T_{i}, \quad X \in \mathscr{L}(H) .
$$

Received by the editors July 1, 1991.

1991 Mathematics Subject Classification. Primary 47A45.

This work was carried out during a visit of the authors at the University of Saarbrücken. The research was supported by the Alexander von Humboldt Foundation, Germany. 
Note that

$$
M_{T}^{s}(X)=\sum_{\substack{\alpha \in \mathbf{Z}_{+}^{n} \\|\alpha|=s}} \frac{s !}{\alpha !} T^{* \alpha} X T^{\alpha}, \quad s=0,1,2, \ldots
$$

Here, as usual, $T^{\alpha}=T_{1}^{\alpha_{1}} \cdots T_{n}^{\alpha_{n}}$ and $T^{*}=\left(T_{1}^{*}, \ldots, T_{n}^{*}\right)$. It is clear that $M_{T}^{s}(X) \geq 0$ whenever $X \geq 0$.

We also define

$$
\Delta_{T}^{(m)}=\left(1-M_{T}\right)^{m}(1), \quad m=0,1,2, \ldots
$$

We have

$$
\Delta_{T}^{(m)}=\sum_{j=0}^{m}(-1)^{j}\left(\begin{array}{c}
m \\
j
\end{array}\right) M_{T}^{j}(1)
$$

and, by (2),

$$
\Delta_{T}^{(m)}=\sum_{|\alpha| \leq m}(-1)^{|\alpha|} \frac{m !}{\alpha !(m-|\alpha|) !} T^{* \alpha} T^{\alpha}
$$

Lemma 1. Let $T \in \mathscr{L}(H)^{n}$ be a commuting multioperator. Then $\Delta_{T}^{(1)} \geq 0$ if and only if $\left\|M_{T}\right\| \leq 1$.

Proof. If $\Delta_{T}^{(1)} \geq 0$ (i.e., $T_{1}^{*} T_{1}+\cdots+T_{n}^{*} T_{n} \leq 1$ ), a direct estimate shows that

$$
\begin{aligned}
\left|\left\langle M_{T}(X) x, y\right\rangle\right| & =\left|\sum_{i=1}^{n}\left\langle X T_{i} x, T_{i} y\right\rangle\right| \leq\|X\| \sum_{i=1}^{n}\left\|T_{i} x\right\|\left\|T_{i} y\right\| \\
& \leq\|X\|\left(\sum_{i=1}^{n}\left\|T_{i} x\right\|^{2}\right)^{1 / 2}\left(\sum_{i=1}^{n}\left\|T_{i} y\right\|^{2}\right)^{1 / 2} \leq\|X\|\|x\|\|y\|
\end{aligned}
$$

for all $X \in \mathscr{L}(H)$ and $x, y \in H$. Hence $\left\|M_{T}\right\| \leq 1$.

Conversely, if $\left\|M_{T}\right\| \leq 1$, then $\left\|M_{T}(1)\right\|=\left\|T_{1}^{*} T_{1}+\cdots+T_{n}^{*} T_{n}\right\| \leq 1$, whence $T_{1}^{*} T_{1}+\cdots+T_{n}^{*} T_{n} \leq 1$.

Lemma 2. Let $T \in \mathscr{L}(H)^{n}$ be a c.m., and let $m \geq 1$ be an integer. The following conditions are equivalent:

(a) $\sup _{s}\left\|M_{T}^{s}(1)\right\|<\infty$ and $\Delta_{T}^{(m)} \geq 0$.

(b) $\Delta_{T}^{(1)} \geq 0$ and $\Delta_{T}^{(m)} \geq 0$.

(c) $\Delta_{T}^{(k)} \geq 0$ for $k=1, \ldots, m$.

Proof. The implication (c) $\Rightarrow(b)$ is clear and the implication (b) $\Rightarrow$ (a) follows from Lemma 1 . We prove $(a) \Rightarrow$ (c).

Suppose condition (a) is satisfied. We may assume $m \geq 2$. Let $x \in H$. For $s=0,1,2, \ldots$ let $a_{s}=\left\langle M_{T}^{s}\left(\Delta_{T}^{(m-1)}\right) x, x\right\rangle$. We have

$$
a_{s}-a_{s+1}=\left\langle M_{T}^{s}\left(1-M_{T}\right)\left(\Delta_{T}^{(m-1)}\right) x, x\right\rangle=\left\langle M_{T}^{s}\left(\Delta^{(m)}\right) x, x\right\rangle \geq 0
$$


as $\Delta_{T}^{(m)} \geq 0$. Thus $a_{s}$ is a nonincreasing sequence of real numbers. Further

$$
\begin{aligned}
\left|\sum_{s=0}^{N} a_{s}\right| & =\left|\sum_{s=0}^{N}\left\langle\left(1-M_{T}\right) M_{T}^{s}\left(\Delta_{T}^{(m-2)}\right) x, x\right\rangle\right| \\
& =\left|\left\langle\Delta_{T}^{(m-2)} x, x\right\rangle-\left\langle M_{T}^{N+1}\left(\Delta_{T}^{(m-2)}\right) x, x\right\rangle\right| \\
& \leq\left(1+\sup _{s}\left\|M_{T}^{s}(1)\right\|\right)\left\|\left(1-M_{T}\right)^{m-2}\right\|\|x\|^{2}<\infty .
\end{aligned}
$$

We conclude that $a_{s} \geq 0$ for every $s$. In particular, $a_{0}=\left\langle\Delta_{T}^{(m-1)} x, x\right\rangle \geq 0$, hence $\Delta_{T}^{(m-1)} \geq 0$.

The assertion (c) now follows by a simple induction.

Corollary 3. If $T \in \mathscr{L}(H)^{n}$ is a c.m. such that $\Delta_{T}^{(1)} \geq 0$ and $\Delta_{T}^{(m)} \geq 0$ for some integer $m \geq 1$, then $0 \leq \Delta_{T}^{(m)} \leq \Delta_{T}^{(m-1)} \leq \cdots \leq \Delta_{T}^{(1)} \leq 1$.

Proof. For $k=1,2, \ldots, m-1$, we have $\Delta_{T}^{(k)}-\Delta_{T}^{(k+1)}=\left(1-M_{T}\right)^{k}(1)-$ $\left(1-M_{T}\right)^{k+1}(1)=M_{T}\left(1-M_{T}\right)^{k}(1) \geq 0$ as $\left(1-M_{T}\right)^{k}(1)=\Delta_{T}^{(k)} \geq 0$ by Lemma 2 .

Lemma 4. Let $T \in \mathscr{L}(H)^{n}$ be a c.m., and let $m \geq 1, N \geq 1$ be integers. Then

$$
\sum_{s=0}^{N}\left(\begin{array}{c}
s+m-1 \\
m-1
\end{array}\right) M_{T}^{s} \Delta_{T}^{(m)} \Delta_{T}^{(m)}=1-\sum_{j=0}^{m-1}\left(\begin{array}{c}
N+j \\
j
\end{array}\right) M_{T}^{N+1} \Delta_{T}^{(j)} .
$$

Proof. We prove the assertion by induction with respect to $m$. If $m=1$, we have

$$
\sum_{s=0}^{N} M_{T}^{s} \Delta_{T}^{(1)}=\sum_{s=0}^{N} M_{T}^{s}\left(1-M_{T}\right)(1)=1-M_{T}^{N+1}(1) .
$$

Now suppose that the assertion holds for $m-1$. Then

$$
\begin{aligned}
& \sum_{s=0}^{N}\left(\begin{array}{c}
s+m-1 \\
m-1
\end{array}\right) M_{T}^{s} \Delta_{T}^{(m)} \\
&=\sum_{s=0}^{N}\left(\begin{array}{c}
s+m-1 \\
m-1
\end{array}\right)\left(1-M_{T}\right) M_{T}^{s} \Delta_{T}^{(m-1)} \\
&= \Delta_{T}^{(m-1)}+\sum_{s=1}^{N}\left[\left(\begin{array}{c}
s+m-1 \\
m-1
\end{array}\right)-\left(\begin{array}{c}
s+m-2 \\
m-1
\end{array}\right)\right] M_{T}^{s} \Delta_{T}^{(m-1)} \\
&-\left(\begin{array}{c}
N+m-1 \\
m-1
\end{array}\right) M_{T}^{N+1} \Delta_{T}^{(m-1)} \\
&= \sum_{s=0}^{N}\left(\begin{array}{c}
s+m-2 \\
m-2
\end{array}\right) M_{T}^{s} \Delta_{T}^{(m-1)}-\left(\begin{array}{c}
N+m-1 \\
m-1
\end{array}\right) M_{T}^{N+1} \Delta_{T}^{(m-1)} \\
&= 1-\sum_{j=0}^{m-1}\left(\begin{array}{c}
N+j \\
j
\end{array}\right) M_{T}^{N+1} \Delta_{T}^{(j)},
\end{aligned}
$$

by the induction hypothesis. 
Lemma 5. Let $T \in \mathscr{L}(H)^{n}$ be a c.m. such that $\Delta_{T}^{(1)} \geq 0$ and $\Delta_{T}^{(m)} \geq 0$ for some integer $m \geq 1$. Let also $x \in H$.

(a) The limit $\lim _{N \rightarrow \infty} N^{k}\left\langle M_{T}^{N}\left(\Delta_{T}^{(k)}\right) x, x\right\rangle$ exists for all $k=0, \ldots, m-1$ and is null for $1 \leq k \leq \max \{1, m-1\}$.

(b) We have $\sum_{s=0}^{\infty} s^{k-1}\left\langle M_{T}^{s}\left(\Delta_{T}^{(k)}\right) x, x\right\rangle<\infty, k=1, \ldots, m$.

Proof. The assertions will be proved simultaneously by induction with respect to $k$. Since $\Delta_{T}^{(1)} \geq 0$ and $M_{T}^{N} \Delta_{T}^{(1)} \geq 0$ for each integer $N \geq 1$, we have

$$
\left\langle M_{T}^{N}(1) x, x\right\rangle-\left\langle M_{T}^{N+1}(1) x, x\right\rangle=\left\langle M_{T}^{N}\left(\Delta_{T}^{(1)}\right) x, x\right\rangle \geq 0 .
$$

Thus $\left\{\left\langle M_{T}^{N}(1) x, x\right\rangle\right\}_{N}$ is a nonincreasing sequence of nonnegative numbers, and so the limit

$$
\lim _{N \rightarrow \infty}\left\langle M_{T}^{N}(1) x, x\right\rangle
$$

exists. Hence (a) holds for $k=0$.

Note also that

$$
\begin{aligned}
& \sum_{s=0}^{\infty}\left\langle M_{T}^{s}\left(\Delta_{T}^{(1)}\right) x, x\right\rangle=\lim _{N \rightarrow \infty} \sum_{s=0}^{N}\left\langle\left(1-M_{T}\right) M_{T}^{s}(1) x, x\right\rangle \\
& \quad=\lim _{N \rightarrow \infty}\left\langle\left(1-M_{T}^{N+1}\right)(1) x, x\right\rangle=\|x\|^{2}-\lim _{N \rightarrow \infty}\left\langle M_{T}^{N+1}(1) x, x\right\rangle<\infty,
\end{aligned}
$$

where the limit exists by (5). Thus (b) holds for $k=1$.

Let us prove now that if (b) holds for some $k, 1 \leq k \leq \max \{1, m-1\}$, and (a) holds for $k-1$, then (a) also holds for $k$. Indeed, if we set $a_{s}=$ $\left\langle M_{T}^{s}\left(\Delta_{T}^{(k)}\right) x, x\right\rangle$, we have $a_{s} \geq 0$ and $a_{s}-a_{s+1}=\left\langle M_{T}\left(\Delta_{T}^{(k+1)}\right) x, x\right\rangle \geq 0$ for all $s$, since $\Delta_{T}^{(k)} \geq 0$ and $\Delta_{T}^{(k+1)} \geq 0$ by Lemma 2 . Thus $\left\{a_{s}\right\}_{s}$ is a nonincreasing sequence of nonnegative numbers satisfying $\sum_{s=0}^{\infty} s^{k-1} a_{s}<\infty$ in virtue of the induction assumption. By [8, Lemma 3.8] it follows that $\lim _{s \rightarrow \infty} s^{k} a_{s}=0$, which shows that (a) holds for $k$.

Conversely, if (a) holds for some $k, 1 \leq k \leq \max \{1, m-1\}$, and (b) holds for $k$, then (b) also holds for $k+1$. Indeed,

$$
\begin{aligned}
\sum_{s=0}^{\infty} s^{k} & \left\langle M_{T}^{s}\left(\Delta_{T}^{(k+1)}\right) x, x\right\rangle \\
= & \lim _{N \rightarrow \infty} \sum_{s=0}^{N} s^{k}\left\langle\left(1-M_{T}\right) M_{T}^{s}\left(\Delta_{T}^{(k)}\right) x, x\right\rangle \\
= & \lim _{N \rightarrow \infty}\left[\sum_{s=1}^{N}\left(s^{k}-(s-1)^{k}\right)\left\langle M_{T}\left(\Delta_{T}^{(k)}\right) x, x\right\rangle-N^{k}\left\langle M_{T}^{N+1}\left(\Delta_{T}^{(k)}\right) x, x\right\rangle\right] \\
& +\left\langle\left(1-M_{T}\right)\left(\Delta_{T}^{(k)}\right) x, x\right\rangle .
\end{aligned}
$$

As $\lim _{N \rightarrow \infty} N^{k}\left\langle M_{T}^{N+1}\left(\Delta_{T}^{(k)}\right) x, x\right\rangle=0$ by the induction hypothesis and $s^{k}-$ $(s-1)^{k} \leq k s^{k-1}$, we infer from (6) that

$$
\sum_{s=0}^{\infty} s^{k}\left\langle M_{T}^{s}\left(\Delta_{T}^{(k+1)}\right) x, x\right\rangle<\infty
$$

via (b) for $k$, which finishes the proof of Lemma 5. 
Let $m$ be a positive integer. We define the function $\rho_{m}: \mathbf{Z}_{+}^{n} \rightarrow \mathbf{Z}_{+}$by the formula

$$
\rho_{m}(\alpha)=\frac{(m+|\alpha|-1) !}{\alpha !(m-1) !}, \quad \alpha \in \mathbf{Z}_{+}^{n} .
$$

Lemma 6. The numbers $\rho_{m}(\alpha)$ satisfy the equation

$$
\sum_{\substack{|\beta| \leq m \\ \beta \leq \alpha}}(-1)^{|\beta|} \frac{m !}{\beta !(m-|\beta|) !} \rho_{m}(\alpha-\beta)=0, \quad \alpha \neq 0 .
$$

Proof. If $\lambda$ is a complex number such that $|\lambda|<1$, then we have the following well-known series expansion

$$
(1-\lambda)^{-m}=\sum_{k=0}^{\infty}\left(\begin{array}{c}
m+k-1 \\
k
\end{array}\right) \lambda^{k}
$$

Therefore, if $z=\left(z_{1}, \ldots, z_{n}\right) \in C^{n}$ satisfies $\sum_{j=1}^{n}\left|z_{j}\right|<1$, we can write

$$
\left(1-\sum_{j=1}^{n} z_{j}\right)^{-m}=\sum_{k=0}^{\infty}\left(\begin{array}{c}
m+k-1 \\
k
\end{array}\right) \sum_{|\gamma| \leq k} \frac{k !}{\gamma !} z^{\gamma}=\sum_{\gamma \geq 0} \rho_{m}(\gamma) z^{\gamma} .
$$

Note also that

$$
\left(1-\sum_{j=1}^{n} z_{j}\right)^{m}=\sum_{|\beta| \leq m}(-1)^{|\beta|} \frac{m !}{\beta !(m-|\beta|) !} z^{\beta} .
$$

By multiplying (9) and (10) we obtain the identity

$$
\sum_{\alpha \geq 0} \sum_{\substack{|\beta| \leq m \\ \beta \leq \alpha}}(-1)^{|\beta|} \frac{m !}{\beta !(m-|\beta|) !} \rho_{m}(\alpha-\beta) z^{\alpha}=1,
$$

whence we easily derive (7).

Consider the Hilbert space $\mathscr{H}=l^{2}\left(\mathbf{Z}_{+}^{n}, H\right)$ consisting of all functions $f: \mathbf{Z}_{+}^{n}$ $\rightarrow H$ such that

$$
\|f\|^{2}=\sum_{\alpha \in \mathbf{Z}_{+}^{n}}|f(\alpha)|^{2}<\infty .
$$

Clearly, $\mathscr{H}$ has a Hilbert space structure with the norm given by (11). We define on the space $\mathscr{H}$ the operators $S_{j}^{(m)}$ by the formula

$$
\left(S_{j}^{(m)} f\right)(\alpha)=\left[\rho_{m}\left(\alpha+e_{j}\right)^{-1} \rho_{m}(\alpha)\right]^{1 / 2} f\left(\alpha+e_{j}\right), \quad j=1, \ldots, n,
$$

where $e_{j}=(0, \ldots, 0,1,0, \ldots, 0) \in \mathbf{Z}_{+}^{n}$ with 1 on the $j$ th position and $m \geq 1$ is a fixed integer. Then $S^{(m)}=\left(S_{1}^{(m)}, \ldots, S_{n}^{(m)}\right) \in \mathscr{L}(H)^{n}$ is a c.m., which is called the backwards multishift of type $(n, m)$.

It is easy to see that $S_{j}^{(m) *}$ is given by

$$
\left(S_{j}^{(m) *} f\right)(\alpha)= \begin{cases}0 & \text { if } \alpha_{j}=0 \\ {\left[\rho_{m}(\alpha)^{-1} \rho_{m}\left(\alpha-e_{j}\right)\right]^{1 / 2} f\left(\alpha-e_{j}\right)} & \text { if } \alpha_{j} \geq 1\end{cases}
$$


Lemma 7. The backwards multishift $S^{(m)}$ has the following properties:

(a) $\Delta_{S^{m}}^{(1)} \geq 0$.

(b) $\Delta_{S^{(m)}}^{(m)} \geq 0$.

(c) $\lim _{s \rightarrow \infty} M_{S^{(m)}}^{s}(1)=0$ in the strong operator topology.

Proof. For every $h \in H$ and $\alpha \in \mathbf{Z}_{+}^{n}$ we denote by $h_{\alpha} \in \mathscr{H}$ the function $h_{\alpha}: \mathbf{Z}_{+}^{n} \rightarrow H$ defined by $h_{\alpha}(\alpha)=h, h_{\alpha}(\beta)=0$ if $\beta \neq \alpha$. The finite sums of elements from the set $\left\{h_{\alpha}, \alpha \in \mathbf{Z}_{+}^{n}, h \in H\right\}$ form a linear subspace dense in $\mathscr{H}$.

Note that for all $h \in H$ and $\alpha \in \mathbf{Z}_{+}^{n}$ we have

$$
S^{(m) \beta} h_{\alpha}= \begin{cases}{\left[\rho_{m}(\alpha)^{-1} \rho_{m}(\alpha-\beta)\right]^{1 / 2} h_{\alpha-\beta}} & \text { if } \beta \leq \alpha, \\ 0 & \text { if } \beta \not \leq \alpha\end{cases}
$$

and, by (13),

$$
S^{(m) * \beta} S^{(m) \beta} h_{\alpha}= \begin{cases}\rho_{m}(\alpha)^{-1} \rho_{m}(\alpha-\beta) h_{\alpha} & \text { if } \beta \leq \alpha, \\ 0 & \text { if } \beta \not \leq \alpha .\end{cases}
$$

Therefore, using (14) for $\beta=e_{j}(j=1, \ldots, n)$,

$$
\begin{aligned}
\Delta_{S^{(m)}}^{(1)} h_{\alpha} & =h_{\alpha}-\sum_{\substack{1 \leq j \leq n \\
\alpha_{j} \neq 0}} \rho_{m}(\alpha)^{-1} \rho_{m}\left(\alpha-e_{j}\right) h_{\alpha} \\
& =\left(1-\sum_{j=1}^{n} \frac{\alpha_{j}}{|\alpha|+m-1}\right) h_{\alpha}=\frac{m-1}{|\alpha|+m-1} h_{\alpha},
\end{aligned}
$$

whence we infer $\Delta_{S^{(m)}}^{(1)} \geq 0$, and so (a) holds.

To prove (b) we observe that if $h \in H$ and $\alpha \in \mathbf{Z}_{+}^{n}, \alpha \neq 0$, then

$$
\begin{aligned}
\Delta_{S^{(m)}}^{(m)} h_{\alpha} & =\sum_{|\beta| \leq m}(-1)^{|\beta|} \frac{m !}{\beta !(m-|\beta|) !} S^{(m) * \beta} S^{(m) \beta} h_{\alpha} \\
& =\sum_{\substack{|\beta| \leq m \\
\beta \leq \alpha}}(-1)^{|\beta|} \frac{m !}{\beta !(m-|\beta|) !} \frac{\rho_{m}(\alpha-\beta)}{\rho_{m}(\alpha)} h_{\alpha}=0,
\end{aligned}
$$

by (14) and Lemma 6 .

If $\alpha=0$, then $\Delta_{S^{(m)}}^{(m)} h_{\alpha}=h_{\alpha}$. Therefore $\Delta_{S^{(m)}}^{(m)} \geq 0$, which establishes (b).

Let again $h \in H$ and $\alpha \in \mathbf{Z}_{+}^{n}$. If $s>|\alpha|$ and $\beta \in \mathbf{Z}_{+}^{n},|\beta|=s$, then $\beta_{j}>\alpha_{j}$ for some $j \in\{1, \ldots, n\}$. Thus $S^{(m) * \beta} S^{(m) \beta} h_{\alpha}=0$ by (14) and hence $M_{S^{(m)}}^{s}(1) h_{\alpha}=0$ by (2). Therefore $\lim _{s \rightarrow \infty} M_{S^{(m)}}^{s}(1) f=0$ for every linear combination of elements of the form $h_{\alpha}$, which is a dense subspace of $\mathscr{H}$. As $\left\|M_{S^{(m)}}^{s}\right\| \leq 1$ for all $s$ by Lemma 1, it follows that (c) also holds.

Let $T \in \mathscr{L}(H)^{n}$ be a c.m. such that $\Delta_{T}^{(1)} \geq 0$ and $\Delta_{T}^{(m)} \geq 0$ for some integer $m \geq 1$. We define an operator $V: H \rightarrow \mathscr{H}$ by the relation

$$
(V h)(\alpha)=\left(\rho_{m}(\alpha) \Delta_{T}^{(m)}\right)^{1 / 2} T^{\alpha} h, \quad h \in H, \alpha \in \mathbf{Z}_{+}^{n} .
$$

Notice that for all $h \in H$ and $\alpha \in \mathbf{Z}_{+}^{n}$ we have

$$
\left(V T_{j} h\right)(\alpha)=\left(\rho_{m}(\alpha) \Delta_{T}^{(m)}\right)^{1 / 2} T^{\alpha+e_{j}} h
$$


and

$$
\left(S_{j}^{(m)} V h\right)(\alpha)=\left[\rho_{m}\left(\alpha+e_{j}\right)^{-1} \rho_{m}(\alpha)\right]^{1 / 2}\left(\rho_{m}\left(\alpha+e_{j}\right) \Delta_{T}^{(m)}\right)^{1 / 2} T^{\alpha+e_{j}} h .
$$

Hence

$$
V T_{j}=S_{j}^{(m)} V, \quad j=1, \ldots, n .
$$

Lemma 8. With the previous notation one has

for every $h \in H$.

$$
\|V h\|^{2}=\|h\|^{2}-\lim _{s \rightarrow \infty}\left\langle M_{T}^{s}(1) h, h\right\rangle
$$

Proof. We have

$$
\begin{aligned}
\|V h\|^{2} & =\sum_{\alpha \in \mathbf{Z}_{+}^{n}} \rho_{m}(\alpha)\left\langle\Delta_{T}^{(m)} T^{\alpha} h, T^{\alpha} h\right\rangle \\
& =\sum_{k=0}^{\infty} \frac{(k+m-1) !}{k !(m-1) !} \sum_{|\alpha|=k} \frac{k !}{\alpha !}\left\langle\Delta_{T}^{(m)} T^{\alpha} h, T^{\alpha} h\right\rangle \\
& =\sum_{k=0}^{\infty}\left(\begin{array}{c}
k+m-1 \\
m-1
\end{array}\right)\left\langle M_{T}^{k}\left(\Delta_{T}^{(m)}\right) h, h\right\rangle \\
& =\lim _{N \rightarrow \infty} \sum_{k=0}^{N}\left(\begin{array}{c}
k+m-1 \\
m-1
\end{array}\right)\left\langle M_{T}^{k}\left(\Delta_{T}^{(m)}\right) h, h\right\rangle \\
& =\|h\|^{2}-\lim _{N \rightarrow \infty} \sum_{j=0}^{m-1}\left(\begin{array}{c}
N+j \\
j
\end{array}\right)\left\langle M_{T}^{N+1}\left(\Delta_{T}^{(j)}\right) h, h\right\rangle \\
& =\|h\|^{2}-\lim _{N \rightarrow \infty}\left\langle M_{T}^{N+1}(1) h, h\right\rangle
\end{aligned}
$$

by Lemmas 4 and 5 .

Theorem 9. Let $T \in \mathscr{L}(H)^{n}$ be a c.m., and let $m \geq 1$ be an integer. The following conditions are equivalent:

(a) $\Delta_{T}^{(1)} \geq 0, \Delta_{T}^{(m)} \geq 0$, and $\lim _{s \rightarrow \infty} M_{T}^{s}(1)=0$ in the strong operator topology.

(b) $T$ is unitarily equivalent to the restriction of $S^{(m)}$ to an invariant subspace. Proof. (a) $\Rightarrow$ (b) Consider the operator $V: H \rightarrow \mathscr{H}$ defined by (15). It follows from Lemma 8 that $V$ is an isometry. Moreover, the space $V H$ is invariant under $S^{(m)}$ by (16), and so (b) holds.

(b) $\Rightarrow$ (a) Let $K \subset \mathscr{H}$ be a (closed, linear) subspace invariant under $S^{(m)}$. If $T_{j}=S_{j}^{(m)} \mid K$ and $x \in K$, it is easily checked that

$$
\left\langle\Delta_{T}^{(k)} x, x\right\rangle=\left\langle\Delta_{S^{(m)}}^{(k)} x, x\right\rangle, \quad k=1,2, \ldots
$$

In particular, $\Delta_{T}^{(1)} \geq 0$ and $\Delta_{T}^{(m)} \geq 0$ by Lemma 7 .

Moreover, if $P_{K}$ is the orthogonal projection of $\mathscr{H}$ onto $K$, as $T^{* \alpha}=$ $P_{K} S^{(m) * \alpha}$ for all $\alpha$, we have

$$
\lim _{s \rightarrow \infty}\left\|M_{T}^{s}(1) x\right\|=\lim _{s \rightarrow \infty}\left\|P_{K} M_{S^{(m)}}^{s}(1) x\right\| \leq \lim _{s \rightarrow \infty}\left\|M_{S^{(m)}}^{s}(1) x\right\|=0,
$$

again by Lemma 7 . This establishes (a).

We recall that a c.m. $T \in \mathscr{L}(H)^{n}$ is said to be spherical isometry if $\Delta_{T}^{(1)}=0$, i.e., $T_{1}^{*} T_{1}+\cdots+T_{n}^{*} T_{n}=1$ (see $\left.[4]\right)$. 
Lemma 10. Let $T \in \mathscr{L}(H)^{n}$ be a c.m. satisfying $\Delta_{T}^{(1)} \geq 0$. Then there exists a Hilbert space $\mathscr{K}$, a spherical isometry $W \in \mathscr{L}(\mathscr{K})^{n}$, and an operator $V_{0}: H \rightarrow$ $\mathscr{K}$ such that $V_{0} T_{j}=W_{j} V_{0}(j=1, \ldots, n)$ and

$$
\left\|V_{0} x\right\|^{2}=\lim _{s \rightarrow \infty}\left\langle M_{T}^{s}(1) x, x\right\rangle
$$

for every $x \in H$.

Proof. We have $1 \geq M_{T}(1) \geq M_{T}^{2}(1) \geq \cdots \geq 0$, so that $\lim _{s \rightarrow \infty}\left\langle M_{T}^{s}(1) x, x\right\rangle$ exists for every $x \in H$.

Define on $H$ the seminorm

$$
\|x\|_{0}^{2}=\lim _{s \rightarrow \infty}\left\langle M_{T}^{s}(1) x, x\right\rangle .
$$

Let $N=\left\{x \in H,\|x\|_{0}=0\right\}$, and let $\mathscr{K}$ be the completion of the quotient $H / N$ with respect to the norm induced by $(17)$, which is clearly a Hilbert space. Let also $V_{0}: H \rightarrow \mathscr{K}$ be given by $V_{0} h=h+N(h \in H)$. Then $\left\|V_{0} x\right\|^{2}=\|x\|_{0}^{2}=$ $\lim _{s \rightarrow \infty}\left\langle M_{T}^{s}(1) x, x\right\rangle$.

Let $W_{1}, \ldots, W_{n}$ be the linear operators defined by

$$
W_{j}(h+N)=T_{j} h+N, \quad h \in H, j=1, \ldots, n .
$$

Since, by (2),

$$
\begin{aligned}
\left\|T_{j} h+N\right\|_{0}^{2} & =\lim _{s \rightarrow \infty}\left\langle M_{T}^{s}(1) T_{j} h, T_{j} h\right\rangle \\
& \leq \lim _{s \rightarrow \infty} \sum_{k=1}^{n}\left\langle M_{T}^{s}(1) T_{k} h, T_{k} h\right\rangle=\lim _{s \rightarrow \infty}\left\langle M_{T}^{s+1}(1) h, h\right\rangle=\|h\|_{0}^{2} .
\end{aligned}
$$

It follows that each $W_{j}$ is correctly defined and it can be continuously extended to the whole space $\mathscr{K}$; this extension will also be denoted by $W_{j}$. Note that $V_{0} T_{j}=W_{j} V_{0}$, which follows from (18).

It remains to prove that $W=\left(W_{1}, \ldots, W_{n}\right)$ is a spherical isometry. Indeed, for every $h \in H$ we have

$$
\begin{aligned}
\sum_{j=1}^{n}\left\|W_{j}(h+N)\right\|_{0}^{2} & =\sum_{j=1}^{n} \lim _{s \rightarrow \infty}\left\langle M_{T}^{s}(1) T_{j} h, T_{j} h\right\rangle \\
& =\sum_{j=1}^{n}\left\langle T_{j}^{*} M_{T}^{s}(1) T_{j} h, h\right\rangle=\left\langle M_{T}^{s+1}(1) h, h\right\rangle,
\end{aligned}
$$

whence

$$
\sum_{j=1}^{n}\left\|W_{j}(h+N)\right\|_{0}^{2}=\|h\|_{0}^{2} .
$$

Theorem 11. Let $T \in \mathscr{L}(H)^{n}$ be a c.m., and let $m \geq 1$ be an integer. The following statements are equivalent:

(a) $\Delta_{T}^{(1)} \geq 0$ and $\Delta_{T}^{(m)} \geq 0$.

(b) $T$ is unitarily equivalent to the restriction of $S^{(m)} \oplus W$ to an invariant subspace, where $W$ is a spherical isometry on some Hilbert space $\mathscr{K}$.

(c) $T$ is unitarily equivalent to the restriction of $S^{(m)} \oplus N$ to an invariant subspace, where $N=\left(N_{1}, \ldots, N_{n}\right)$ and $N_{1}, \ldots, N_{n}$ are commuting normal operators on a Hilbert space $\mathscr{K}^{\prime}$ satisfying $N_{1}^{*} N_{1}+\cdots+N_{n}^{*}=1$. 
Proof. (a) $\Rightarrow$ (b) Let $V: H \rightarrow \mathscr{H}$ be the operator defined by (15) and let $\mathscr{K}, V_{0}: H \rightarrow \mathscr{K}$ and $W \in \mathscr{L}(\mathscr{K})^{n}$ be given by Lemma 10 . If we set $V_{1} h=$ $V h \oplus V_{0} h$ for each $h \in H$, then $V_{1}: K \rightarrow \mathscr{H} \oplus \mathscr{K}$ is an isometry, which satisfies $V_{1} T^{\alpha} h=\left(S^{(m) \alpha} \oplus W^{\alpha}\right) V_{1} h$ for all $\alpha \in Z_{+}^{n}$ and $h \in H$ by (18) and Lemmas 8 and 10 .

(b) $\Rightarrow$ (c) By a result of Athavale [4], the spherical isometry $W \in \mathscr{L}(K)^{n}$ has an extension $N=\left(N_{1}, \cdots, N_{n}\right) \in \mathscr{L}\left(\mathscr{K}^{\prime}\right)^{n}$, where $\mathscr{K}^{\prime}$ is a Hilbert space containing $\mathscr{K}, N_{1}, \ldots, N_{n}$ are commuting normal operators on $\mathscr{K}^{\prime}$ satisfying $N_{1}^{*} N_{1}+\cdots+N_{n}^{*} N_{n}=1$, and $N_{j} \mid H=W_{j}(j=1, \ldots, n)$. This gives (c).

(c) $\Rightarrow$ (a) We have $\Delta_{S^{(m)}}^{(1)} \geq 0$ and $\Delta_{S^{(m)}}^{(m)} \geq 0$ by Lemma 8 . Further $\Delta_{N}^{(1)}=0$, and so $\Delta_{N}^{(m)}=\left(1-M_{N}\right)^{m-1} \Delta_{N}^{(1)}=0$. Thus $\Delta_{S^{(m)} \oplus N}^{(1)} \geq 0$ and $\Delta_{S^{(m)} \oplus N}^{(m)} \geq 0$. As these inequalities remain true for any restriction of $S^{(m)} \oplus N$ to an invariant subspace, (a) is established.

Remarks. $1^{\circ}$. The case $m=n=1$. This is the classical Sz.-Nagy-Foias dilation theory [9]. In this case, $\Delta_{T}^{(1)}=1-T_{1}^{*} T_{1}$ is the square of the defect operator and $\Delta_{T}^{(1)} \geq 0$ holds if and only if $\left\|T_{1}\right\| \leq 1$. The condition $\lim _{s \rightarrow \infty} M_{T}^{s}(1) x=0$ for every $x \in H$ reduces in this case to the $C_{0}$ condition $\lim _{s \rightarrow \infty} T_{1}^{s} x=0(x \in H)$.

$2^{\circ}$. The case $n=1, m \geq 2$. This is the case of $m$-hypercontractions of Agler [1] (cf. also [8]).

$3^{\circ}$. The case $n \geq 2, m=1$. This case has been studied by Drury [7] (see also [5]).

$4^{\circ}$. The case $m=n$. This case has been studied in [10], (see also [4]), and is particularly interesting since one has the additional property $\Delta_{S^{(n) *}}^{(1)}=0$, i.e., $S^{(n) *}$ is a spherical isometry. Indeed, if $h \in H$ and $\alpha \in \mathbf{Z}_{+}^{n}$, then, with the notation of Lemma 8,

$$
\sum_{j=1}^{n} S_{j}^{(n)} S_{j}^{(n) *} h_{\alpha}=\sum_{j=1}^{n} \frac{\rho_{n}(\alpha)}{\rho_{n}\left(\alpha+e_{j}\right)} h_{\alpha}=\left(\sum_{j=1}^{n} \frac{\alpha_{j}+1}{|\alpha|+n}\right) h_{\alpha}=h_{\alpha},
$$

and so the same equality holds for all $f \in \mathscr{H}$. By the above-mentioned result of Athavale [4], it follows from Theorem 11 that every c.m. $T \in \mathscr{L}(H)^{n}$ satisfying $\Delta_{T}^{(1)} \geq 0$ and $\Delta_{T}^{(n)} \geq 0$ has a spherical dilation, i.e., there exist a Hilbert space $K \supset H$ and a c.m. $N=\left(N_{1}, \ldots, N_{n}\right) \in \mathscr{L}(K)^{n}$ consisting of normal operators such that $N_{1}^{*} N_{1}+\cdots+N_{n}^{*} N_{n}=1$ on $K$ and $T^{\alpha}=P_{H} N^{\alpha} \mid H$ for all $\alpha \in Z_{+}^{n}$, where $P_{H}$ is the orthogonal projection of $K$ onto $H$. So in this case a complete analogy with the Sz.-Nagy-Foias theory might be developed (see [10]).

In particular, for a c.m. $T \in \mathscr{L}(H)^{n}$ such that $\Delta_{T}^{(1)} \geq 0, \Delta_{T}^{(n)} \geq 0$, and $\lim _{s \rightarrow \infty} M_{T}^{s}(1) x=0, x \in H$, we derive the existence of a contractive unital algebra homomorphism $\Phi_{T}: H^{\infty}(B) \rightarrow \mathscr{L}(H)$ as in Theorem 4.20 from [10].

$5^{\circ}$. If $m=n+1$, then the multishift operator $S_{j}^{(m) *}(j=1, \ldots, n)$ is unitarily equivalent to the multiplication by the variables $z_{j}(j=1, \ldots, n)$ on the Bergman space. The Bergman space consists of all analytic functions in the open unit ball $B$ of $C^{n}$, which lie in $L^{2}(B, d \nu)$, where $\nu$ is the normalized Lebesgue measure. 
Analogously, for $m>n, S_{j}^{(m) *}(j=1, \ldots, n)$ is unitarily equivalent to the multiplication by $z_{j}$ in the weighted Bergman space, which consists of all analytic functions in $B$ that lie in $L^{2}(B, d \mu)$, where $d \mu=c_{m}\left(1-|z|^{2}\right)^{m-n-1} d \nu(z)$ and $c_{m}$ is a normalization constant chosen such that $\mu(B)=1$ (see, e.g., [2, 5]).

$6^{\circ}$. Condition $\Delta_{T}^{(1)} \geq 0$ is necessary but not sufficient to ensure the existence of a spherical dilation for a c.m. $T \in \mathscr{L}(H)^{n}$ if $n \geq 2$ (cf. also [7]). Indeed, with $K$ and $N$ as in $4^{\circ}$, we have

$$
\sum_{j=1}^{n}\left\langle T_{j}^{*} T_{j} x, x\right\rangle=\sum_{j=1}^{n}\left\langle P_{H} N_{j} x, P_{H} N_{j} x\right\rangle \leq \sum_{j=0}^{n}\left\langle N_{j} x, N_{j} x\right\rangle=\|x\|^{2}
$$

for each $x \in H$, i.e., $\Delta_{T}^{(1)} \geq 0$. Similarly, $\Delta_{T^{*}}^{(1)} \geq 0$. On the other hand, if $1 \leq m<n$, with $S^{(m)}, h$, and $h_{\alpha}$ as in Lemma 7, one deduces from (12) and (13) that

$$
\begin{aligned}
\left\langle\Delta_{S^{(m) *}}^{(1)} h_{\alpha}, h_{\alpha}\right\rangle & =\left(1-\sum_{j=1}^{n} \frac{\rho_{m}(\alpha)}{\rho_{m}\left(\alpha+e_{j}\right)}\right)\|h\|^{2} \\
& =\left(1-\sum_{j=1}^{n} \frac{\alpha_{j}+1}{m+|\alpha|}\right)\|h\|^{2}=\frac{m-n}{|\alpha|+m}\|h\|^{2}<0
\end{aligned}
$$

if $h \neq 0$. Hence $S^{(m)}$ cannot have a spherical dilation.

$7^{\circ}$. If $\Delta_{T}^{(1)} \geq 0$, then the joint (Taylor) spectrum $\sigma(T)$ is contained in the closed unit ball of $\bar{B}$ of $\mathbf{C}^{n}$. To prove this, denote by

$$
\sigma_{\pi}(T)=\left\{\lambda \in \mathbf{C}^{n}, \text { inf }\left\{\sum_{i=1}^{n}\left\|\left(T_{i}-\lambda_{i}\right) x\right\|, x \in H,\|x\|=1\right\}=0\right\}
$$

the joint approximate point spectrum of $T$.

If $\lambda \in \sigma_{\pi}(T)$, then there exists a sequence $\left\{x_{k}\right\}_{k} \subset H,\left\|x_{k}\right\|=1$ with $\lim _{k \rightarrow \infty}\left(T_{i}-\lambda_{i}\right) x_{k}=0(i=1, \ldots, n)$ and $1=\left\|x_{k}\right\| \geq \sum_{i=1}^{n}\left\|T_{i} x_{k}\right\|^{2} \rightarrow$ $\sum_{i=1}^{n}\left|\lambda_{i}\right|^{2}$. So $\sigma_{\pi}(T) \subset \bar{B}$ and, by [6], $\sigma(T) \subset \bar{B}$ (this is true for any reasonable joint spectrum).

\section{REFERENCES}

1. J. Agler, Hypercontractions and subnormality, J. Operator Theory 13 (1985), 203-217.

2. J. Arazy, S. D. Fisher, S. Janson, and J. Peetre, Membership of Hankel operators on the ball in unitary ideals, preprint, Upsala, 1989.

3. A. Athavale, Holomorphic kernels and commuting operators, Trans. Amer. Math. Soc. 304 (1987), 101-110.

4. __, On the intertwining of joint isometries, J. Operator Theory 23 (1990), 339-350.

5. _ Model theory on the unit ball in $\mathbf{C}^{n}$, J. Operator Theory (to appear).

6. M. Cho and W. Żelazko, On geometric spectral radius of commuting $n$-tuples of operators, Hokkaido Math. J. (to appear)

7. S. W. Drury, A generalization of von Neumann's inequality to complex ball, Proc. Amer. Math. Soc. 68 (1978), 300-304.

8. V. Müller, Models for operators using weighted shifts, J. Operator Theory 20 (1988), 3-20. 
9. B. Sz.-Nagy and C. Foiaş, Analyse harmonique des opérateurs de l' espace de Hilbert, Masson, Paris, and Akad. Kiadó, Budapest, 1967.

10. F.-H. Vasilescu, An operator-valued Poisson kernel, preprint.

Institute of Mathematics, Czechoslovak Academy of Sciences, Žitná 25, 11567 Praha 1, Czechoslovakia

E-mail address: MULLER@CSPGAS11.bitnet

Institute of Mathematics of the Romanian Academy, P.O. Box 1-764, RO-707001 BuchAREST, ROMANIA 